\title{
Asymptotic Computation of the Repeated Integrals of the Error Function Complement
}

\author{
By W. R. Wilcox
}

Abstract. Previously, the complementary error function and its repeated integrals were given only for small values of the argument. Several new calculation techniques are derived which permit evaluation for the complete range of the argument. Some new values of these functions for large values of the argument are calculated. These values are plotted in such a manner that approximate values can easily be found for all values of $x$.

1. Introduction. The error function and its repeated integrals occur frequently in solutions to one-dimensional heat transfer and mass transfer problems. The error function is defined by

$$
\operatorname{erf} x \equiv \frac{2}{\sqrt{\pi}} \int_{0}^{x} e^{-\epsilon^{2}} d \epsilon
$$

and the complement of the error function by

$$
\operatorname{erfc} x \equiv 1-\operatorname{erf} x=\frac{2}{\sqrt{\pi}} \int_{x}^{\infty} e^{-\epsilon^{2}} d \epsilon
$$

The repeated integrals of the error function complement are defined by*

$$
i^{n} \operatorname{erfc} x=\int_{x}^{\infty} i^{n-1} \operatorname{erfc} \epsilon d \epsilon, \quad \text { for } n=1,2, \cdots
$$

with

$$
i^{0} \operatorname{erfc} x=\operatorname{erfc} x
$$

Hartree [1] observed that $y=i^{n} \operatorname{erfc} x$ satisfies the differential equation

$$
\frac{d^{2} y}{d x^{2}}+2 x \frac{d y}{d x}-2 n y=0
$$

The repeated integrals of the error function complement also occur in solutions to problems in heat and mass transfer in which the conditions on the boundary of a semi-infinite body are given as a function of time $[2,3]$. This follows from the fact that $y=t^{n / 2} i^{n} \operatorname{erfc}\left[x /(4 D t)^{1 / 2}\right]$ satisfies the differential equation

$$
\frac{\partial^{2} y}{\partial x^{2}}-\frac{1}{D} \frac{\partial y}{\partial t}=0
$$

2. Techniques of Computation. Previously [1, 4, 5-8], values for the complementary error function and its repeated integrals were given only for small values

Received February 4, 1963. Revised June 7, 1963.

* In words, $i^{n} \operatorname{erfc} x$ denotes $n$ repeated integrations from $x$ to $\infty$ of the error function complement of $x$. 
of the argument*. These prior evaluations were made by means of variations of the general recurrence formula:

$$
2 n i^{n} \operatorname{erfc} x=i^{n-2} \operatorname{erfc} x-2 x i^{n-1} \operatorname{erfc} x
$$

for $n \geqq 2$, with

$$
i \operatorname{erfc} x=\frac{1}{\sqrt{\pi}} \exp \left(-x^{2}\right)-x \operatorname{erfc} x .
$$

Extensive tables for $\left(\frac{2}{\sqrt{\pi}}\right) \exp \left(-x^{2}\right)$ and for erf $x$ are given by the National Bureau of Standards [9].

The difficulty with this technique is that each successive integral is found by the difference of functions of the preceding two integrals. As this process is repeated significant figures are rapidly lost. As a result the calculation is soon brought to a halt as $x$ and $n$ increase, even when a modern digital computer is used. If Equation (2) is used along with the tables of erf $x$ given in Ref. 9 to calculate erfc $x$ a similar problem is encountered because erf $x$ rapidly approaches unity as $x$ increases.

This paper describes a series approximation which becomes more accurate as $x$ increases. Carslaw and Jaeger [2] give the following asymptotic series for erfc with large $x$ :

$$
\text { erfc } x \cong \frac{\exp \left(-x^{2}\right)}{x \sqrt{\pi}}\left[1-\frac{1}{2 x^{2}}+\frac{1 \cdot 3}{2^{2} x^{4}}-\frac{1 \cdot 3 \cdot 5}{2^{3} x^{6}}+\cdots\right]
$$

Since this series is not convergent it is terminated at the first term which is larger than the previous one. Substitution of Equation (9) into Equation (8) yields

$$
i \text { erfc } x \cong \frac{1}{2 \sqrt{ } \pi} \frac{\exp \left(-x^{2}\right)}{x^{2}}\left[1-\frac{1 \cdot 3}{2 x^{2}}+\frac{1 \cdot 3 \cdot 5}{2^{2} x^{4}}-\frac{1 \cdot 3 \cdot 5 \cdot 7}{2^{3} x^{6}}+\cdots\right] \text {. }
$$

This enables calculation of $i$ erfe $x$ for large $x$. In a like manner it can be shown that

$$
i^{n} \operatorname{erfc} x \cong \frac{2 \exp \left(-x^{2}\right)}{\sqrt{\pi}} \frac{1}{(2 x)^{n+1}}\left[1+\sum_{i=1}^{i_{a}}(-1)^{i} \frac{r_{i, n}}{2^{i} x^{2 i}}\right]
$$

A general formula for $r_{i, n}$ is found by substitution of Equation (11) into Equation (7), equating terms of like powers of $x$, and applying the results repeatedly, to be

$$
r_{i, n}=1 \cdot 3 \cdot 5 \cdot \cdots \cdot(2 i-1)(n+2 i)_{2 i},
$$

where $(n+2 i)_{2 i}$ is a binomial coefficient.

Similar asymptotic expressions for $x<1$ for the repeated integrals may be found by use of Taylor's series for erf $x$ and $\exp \left(-x^{2}\right)$ with repeated application of the recurrence equation (7). Values of the repeated integrals for $x=0$ are found by repeated application of Eq. (7) to be [1]:

$$
i^{n} \operatorname{erfc} 0=\frac{1}{2^{n}\left(\frac{n}{2}\right) !}=\frac{1}{2^{n} \Gamma\left(\frac{n}{2}+1\right)} .
$$

* The British Association Mathematical Tables [5] actually give tabulations of $H h$ (Herm itian probability) functions. Values of $i^{n} \operatorname{erfc}(x)$ are obtainable from the relationship

$$
i^{n} \operatorname{erfc}(x)=2^{-\frac{i}{2} n}(2 / \pi)^{\frac{1}{2}} H h_{n}(x \sqrt{2})
$$




\begin{tabular}{|c|c|c|c|c|c|c|c|c|}
\hline & Tabulation o & $\frac{(2 x)^{n+1} \sqrt{ }}{2 \exp }$ & $\frac{\pi i^{n} \operatorname{erfc} x}{\left(-x^{2}\right)}$ or & $1+\sum_{i=1}^{1=i_{c}}$ & $(-1)^{i} \frac{r_{i, n}}{2^{i} x^{2 i}}$ & as $a F_{2}$ & unction of $x^{*} a$ & $n d n$ \\
\hline$x$ & $n=0$ & & $n=1$ & & $n=2$ & & $n=3$ & \\
\hline $\begin{array}{l}0 \\
.01 \\
.1 \\
.5 \\
1 \\
1.5 \\
2 \\
2.5 \\
3 \\
3\end{array}$ & \begin{tabular}{l}
\multicolumn{1}{c}{ Kaye } \\
\multicolumn{1}{c}{0} \\
0.01753 \\
0.1589 \\
0.5456 \\
0.7262 \\
0.8539 \\
0.9054 \\
0.934
\end{tabular} & $\begin{array}{l}0.313 \\
0.844 \\
0.904 \\
0.934 \\
0.951 \\
0.971 \\
0.981\end{array}$ & $\begin{array}{c}\text { Kaye } \\
0 \\
1.965 \stackrel{1}{\times} 10^{-4} \\
0.01682 \\
0.2272 \\
0.4842 \\
0.6528 \\
0.7570\end{array}$ & $\begin{array}{l}0.250 \\
0.505 \\
0.726 \\
0.821 \\
0.866 \\
0.918 \\
0.945\end{array}$ & \begin{tabular}{|c|c} 
& Kaye \\
0 \\
$1.733 \times 10^{-6}$ \\
$5.004 \times 10^{-3}$ \\
0.07962 \\
0.2736 \\
0.4558 \\
0.5913 \\
\\
\end{tabular} & \begin{tabular}{|l}
-0.500 \\
0.333 \\
0.543 \\
0.684 \\
0.760 \\
0.847 \\
0.8952
\end{tabular} & $\begin{array}{c}\text { Kaye } \\
0 \\
1.298 \stackrel{1}{\times} 10^{-8} \\
1.027 \times 10^{-4} \\
0.02459 \\
0.1404 \\
0.2956 \\
0.4384\end{array}$ & $\begin{array}{r}-1.50 \\
-0.111 \\
0.375 \\
0.549 \\
0.610 \\
0.771 \\
0.837\end{array}$ \\
\hline$x$ & $n=4$ & & $n=5$ & & $n=6$ & & $n=7$ & \\
\hline $\begin{array}{l}0 \\
.01 \\
.1 \\
.5 \\
1 \\
1.5 \\
2 \\
2.5 \\
3\end{array}$ & $\begin{array}{c}\text { Kaye } \\
0 \\
8.597 \times 10^{-11} \\
6.590 \times 10^{-6} \\
6.878 \times 10^{-3} \\
0.06656 \\
0.1801 \\
(0.33) \dagger \\
(0.42)\end{array}$ & $\begin{array}{l}-2.75 \\
-0.666 \\
0.0625 \\
0.400 \\
0.519 \\
0.672 \\
0.765\end{array}$ & $\begin{array}{c}\text { Kaye } \\
0 \\
5.156 \times 10^{-13} \\
3.842 \times 10^{-7} \\
1.772 \times 10^{-3} \\
0.0295 \\
0.1039 \\
(0.22) \\
(0.32) \\
(0.42)\end{array}$ & \begin{tabular}{|c|}
-4.25 \\
-1.33 \\
-0.312 \\
0.160 \\
0.417 \\
0.581 \\
0.691
\end{tabular} & $\begin{array}{c}\text { Kaye } \\
0 \\
2.848 \times 10^{-15} \\
2.068 \times 10^{-8} \\
4.255 \times 10^{-4} \\
0.01235 \\
0.05721 \\
(0.14) \\
(0.22) \\
(0.33)\end{array}$ & $\mid \begin{array}{l}-6.00 \\
-2.11 \\
-0.750 \\
-0.120 \\
0.222 \\
0.491 \\
0.616\end{array}$ & $\begin{array}{c}\text { Kaye } \\
0 \\
1.465 \times 10^{-17} \\
1.039 \times 10^{-9} \\
9.615 \times 10^{-5} \\
4.903 \times 10^{-3} \\
0.03008 \\
(0.083) \\
(0.15) \\
(0.26) \\
(0.41)\end{array}$ & $\begin{array}{r}-8.00 \\
-3.00 \\
-1.25 \\
-0.440 \\
0.000 \\
0.438 \\
0.542\end{array}$ \\
\hline
\end{tabular}

* Values of $F(n, x)$ calculated from Kaye's data [4] for $i^{n}$ erf $c x$. Values of $\left[1+\sum\right]$ as calculated in this paper. Present asymptotic calculation technique is valid when these two values are approximately equal.

$\dagger$ Note: Numbers in parenthesis were derived by extrapolation in Figure 1.

The foregoing provides the means to calculate $i^{n}$ erfc a for both high and low values of $x$ without taking repeated differences. Finally, from the standpoint of completeness, it is of some interest to reexamine the relation given by equation ( 7 ). If we apply equation (7) repeatedly we obtain the following:

$$
\begin{aligned}
i^{n} \operatorname{erfc} x & =-\frac{x}{n} i^{n-1} \operatorname{erfc} x+\frac{i^{n-2} \operatorname{erfc} x}{2 n} \\
& =i^{n-2} \operatorname{erfc} x\left(\frac{1}{2 n}+\frac{x^{2}}{n(n-1)}\right)-i^{n-3} \operatorname{erfc} x\left(\frac{x}{2 n(n-1)}\right) \\
& =i^{n-3} \operatorname{erfc} x\left[x\left(\frac{1}{2 n(n-2)}+\frac{1}{2 n(n-1)}\right)+\frac{x^{3}}{n(n-1)(n-2)}\right] \\
& \quad+\frac{i^{n-4} \operatorname{erfc} x}{2(n-2)}\left(\frac{1}{2 n}+\frac{x^{2}}{n(n-1)}\right) \\
& =\text { etc. }
\end{aligned}
$$

In this way each $i^{n}$ erfc $x$ can be related clear back to a single difference between functions of erfe $x$ and $i \operatorname{erfc} x$. As $x$ decreases and $n$ increases these expressions 


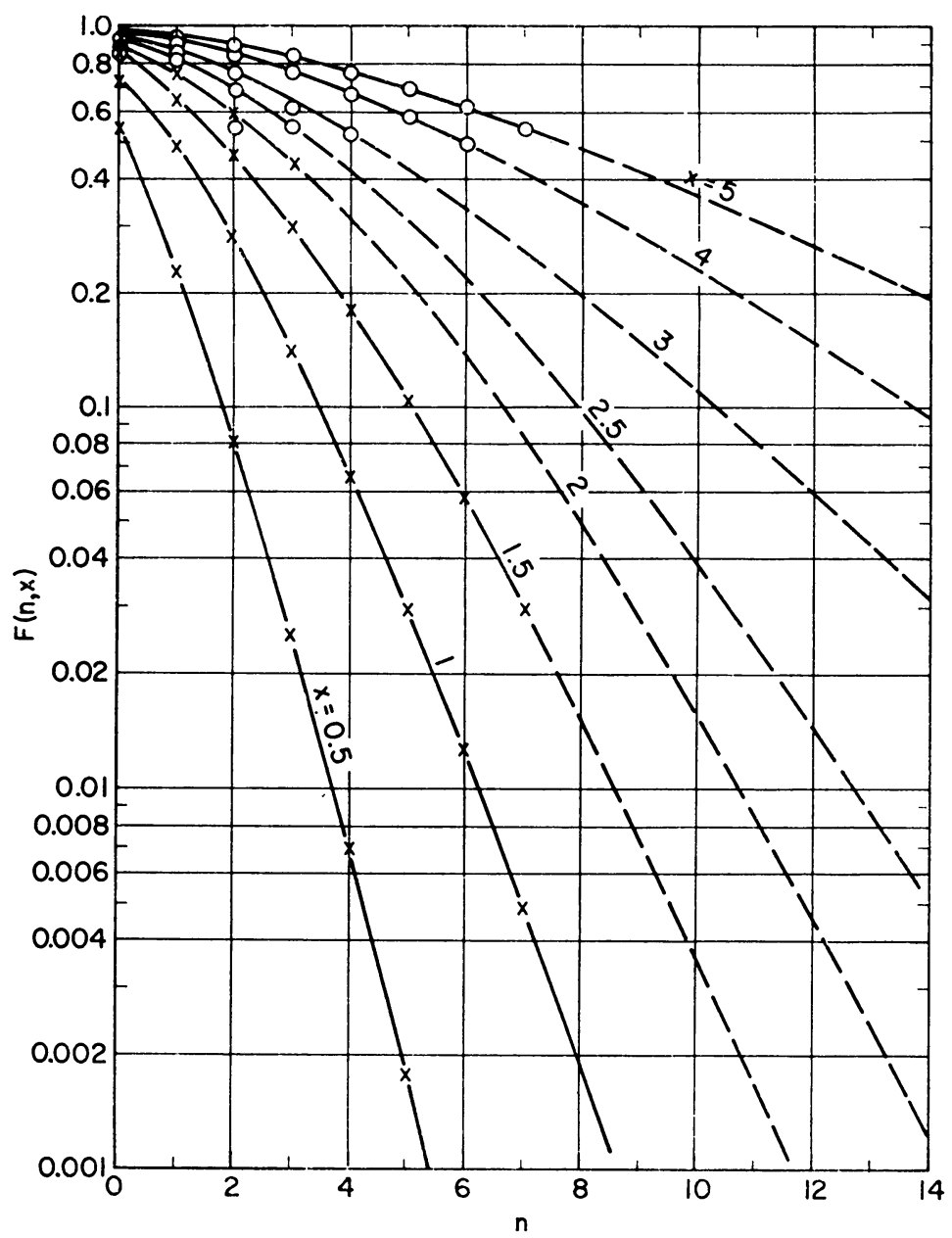

Fig. 1. $\frac{(2 x)^{n+1} \sqrt{\pi} i^{n} \operatorname{erfc} x}{2 \exp \left(-x^{2}\right)}$ As a Function of $n$ for Various Values of $x . x$-values from Kaye [4]. 0-values of $\left(1+\sum\right)$ from this paper.

become increasingly accurate and do not depend on repeated difference operations, so that a respectable number of significant figures can be obtained.

3. Numerical Values. As shown in Table 2 and Figure 3, Kaye [4] has calculated $i^{n}$ erfc $x$ for small values of $x$ using the repeated difference method of equation (7). The present work was intended to extend these results to higher values of $x$. The calculations were performed on a desk calculator. The infinite series method of equation (11) was used. As noted previously, these series are not convergent. Consequently, in obtaining approximate values for $\left[1+\sum\right]^{*}$, the summation was stopped at the first negative term ( $i_{c}$ odd) for which the value is greater than the

$* \sum$ is used in place of $\sum_{i=1}^{i_{c}}(-1)^{i} \frac{r_{i, n}}{2^{i} x^{2 i}}$ to save space. 


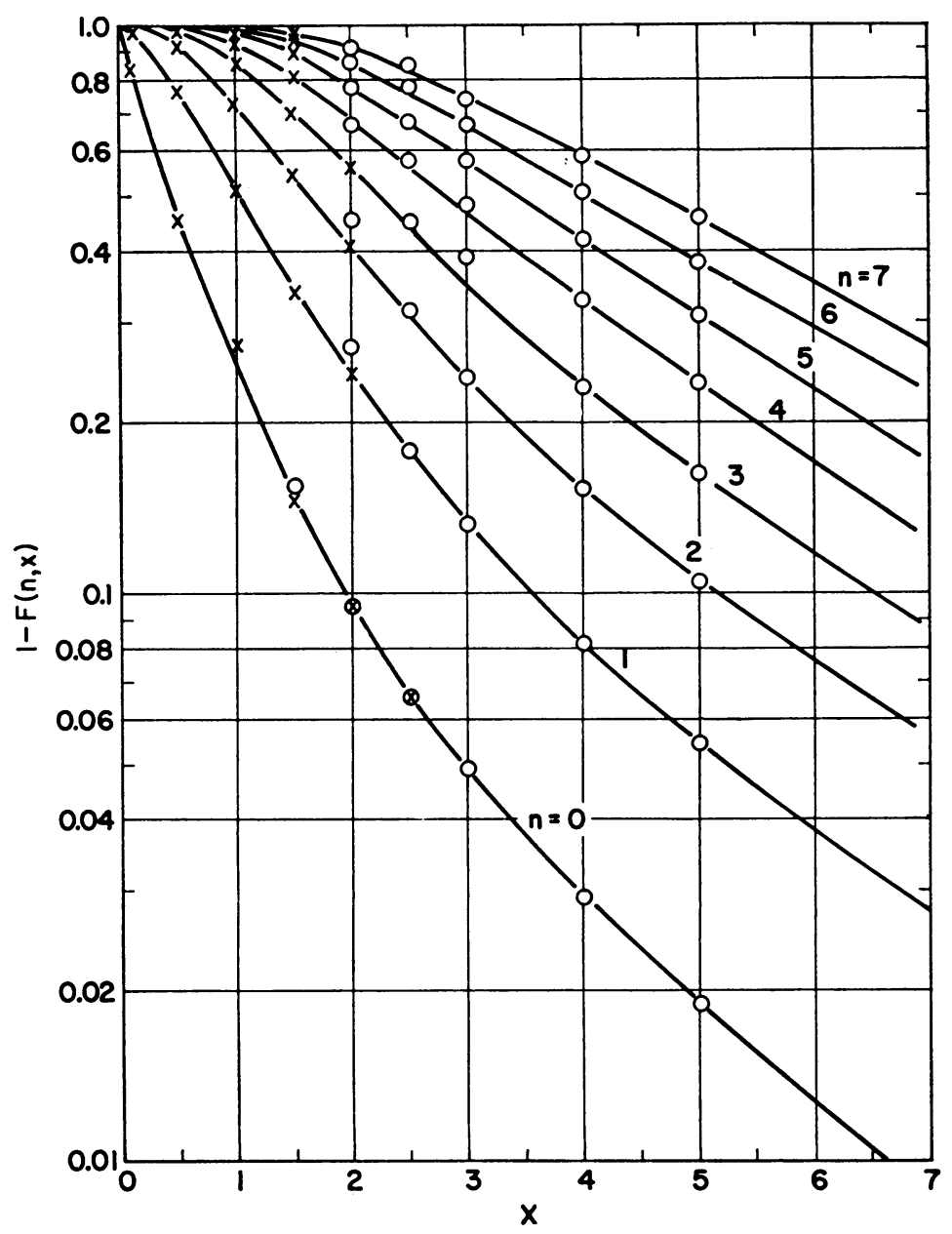

Fig. 2. $\left[1-\frac{(2 x)^{n+1} \sqrt{\pi} i^{n} \text { erfc } x}{2 \exp \left(-x^{2}\right)}\right]$ As a Function of $x$ for Various Values of $n$. $x$-values from Kaye [4]. $0-$ values of $-\sum$ from this paper.

previous negative term. In addition, only half of the value of this last term was used, since comparison with Kaye's results, where possible, indicated that this method gave better results. As $x$ increased, the relative value of this final term decreased, and so the approximation became increasingly accurate. The calculated values for the summations are given in Table 1 . It is seen that the minimum value of $x$ for which this approximate technique works increases with increasing $n$. To the first approximation this minimum value of $x$ is given by the condition that the first term, $r_{1, n} / 2 x^{2}$, be less than 1 , or that $x>\sqrt{r_{1, n} / 2}=\sqrt{(n+2)_{2}} / \sqrt{2}$. The values in parentheses in Table 1 were obtained by extrapolation of plots of $F(n, x)^{*}$ as a function of $n$ for constant $x$, as shown in Figure 1. From Figure 1

* $F(n, x)$ is used in place of $\frac{(2 x)^{n+1} \sqrt{ } \pi i^{n} \operatorname{erfc} x}{2 \exp \left(-x^{2}\right)}$ to save space. 
TABLE 2

Repeated Integrals of the Error Function, $i^{n}$ erfc $x^{*}$

\begin{tabular}{|c|c|c|c|c|c|c|}
\hline$x$ & \multicolumn{2}{|c|}{$\operatorname{erfc} x$} & \multicolumn{2}{|c|}{$i \operatorname{erfc} x$} & \multicolumn{2}{|c|}{$i^{2} \operatorname{erfc}$} \\
\hline $\begin{array}{l}0 \\
\quad .01 \\
.1 \\
.5 \\
1 \\
1.5 \\
2 \\
2.5 \\
3 \\
4 \\
5\end{array}$ & \begin{tabular}{l}
\multicolumn{1}{c}{ Kaye } \\
1.000 \\
0.9887 \\
0.8875 \\
0.4795 \\
0.1573 \\
0.03390 \\
$4.678 \times 10^{-3}$ \\
$4.07 \times 10^{-4}$
\end{tabular} & $\begin{array}{c}0.0334 \\
4.67 \times 10^{-3} \\
4.07 \times 10^{-4} \\
2.21 \times 10^{-5} \\
1.54 \times 10^{-8} \\
1.57 \times 10^{-12}\end{array}$ & $\begin{array}{l}\quad \text { Kaye } \\
0.5642 \\
0.5542 \\
0.4698 \\
0.1996 \\
0.05026 \\
8.623 \times 10^{-8} \\
9.78 \times 10^{-4}\end{array}$ & $\begin{array}{c}\text { Present } \\
\\
\\
9.38 \times 10^{-4} \\
7.15 \times 10^{-5} \\
3.35 \times 10^{-6} \\
1.82 \times 10^{-9} \\
1.48 \times 10^{-13}\end{array}$ & \begin{tabular}{l}
\multicolumn{1}{|c}{ Kaye } \\
0.2500 \\
0.2444 \\
0.1984 \\
0.06997 \\
0.01420 \\
$2.007 \times 10^{-3}$ \\
$1.91 \times 10^{-4}$
\end{tabular} & $\begin{array}{l}1.75 \times 10^{-4} \\
1.19 \times 10^{-5} \\
4.90 \times 10^{-7} \\
2.10 \times 10^{-10} \\
1.40 \times 10^{-14}\end{array}$ \\
\hline$x$ & \multicolumn{2}{|c|}{$i^{3} \operatorname{erfc} x$} & \multicolumn{2}{|c|}{$i^{4} \operatorname{erfc} x$} & \multicolumn{2}{|c|}{$i^{5} \operatorname{erfc} x$} \\
\hline $\begin{array}{l}0 \\
\quad .01 \\
.1 \\
.5 \\
1 \\
1.5 \\
2 \\
2.5 \\
3 \\
4 \\
5\end{array}$ & \begin{tabular}{l}
\multicolumn{1}{c}{ Kaye } \\
0.09403 \\
0.09156 \\
0.07169 \\
0.02161 \\
$3.643 \times 10^{-3}$ \\
$4.339 \times 10^{-4}$ \\
$3.54 \times 10^{-5}$
\end{tabular} & $\begin{array}{l}1.91 \times 10^{-6} \\
6.56 \times 10^{-8} \\
2.39 \times 10^{-11} \\
1.31 \times 10^{-15}\end{array}$ & \begin{tabular}{l}
\multicolumn{1}{c}{ Kaye } \\
0.03125 \\
0.03032 \\
0.02301 \\
$6.044 \times 10^{-3}$ \\
$8.639 \times 10^{-4}$ \\
$8.81 \times 10^{-5}$
\end{tabular} & $\begin{array}{c}\text { Present } \\
\\
\left(6.7 \times 10^{-6}\right) \dagger \\
\left(2.9 \times 10^{-7}\right) \\
9.43 \times 10^{-9} \\
2.61 \times 10^{-12} \\
1.20 \times 10^{-16}\end{array}$ & $\begin{array}{c}\text { Kaye } \\
9.403 \times 10^{-3} \\
9.095 \times 10^{-3} \\
6.709 \times 10^{-3} \\
1.557 \times 10^{-3} \\
1.916 \times 10^{-4} \\
1.695 \times 10^{-5}\end{array}$ & $\begin{array}{l}\left(1.1 \times 10^{-6}\right) \\
\left(4.5 \times 10^{-8}\right) \\
\left(1.3 \times 10^{-9}\right) \\
2.81 \times 10^{-13} \\
1.08 \times 10^{-17}\end{array}$ \\
\hline$x$ & \multicolumn{3}{|c|}{$i^{6} \operatorname{erfc} x$} & \multicolumn{3}{|c|}{$i^{7} \operatorname{erfc} x$} \\
\hline $\begin{array}{l}0 \\
\quad .01 \\
.1 \\
.5 \\
1 \\
1.5 \\
2 \\
2.5 \\
3 \\
4 \\
5\end{array}$ & $\begin{array}{l}\quad K a ? \\
2.604 \times \\
2.512 \times \\
1.805 \times \\
3.739 \times \\
4.007 \times \\
3.11 \times\end{array}$ & $\begin{array}{l}10^{-3} \\
10^{-3} \\
10^{-3} \\
10^{-4} \\
10^{-5} \\
10^{-6}\end{array}$ & $\begin{array}{l}\left(1.7 \times 10^{-7}\right) \\
\left.6.0 \times 10^{-9}\right) \\
\left(1.6 \times 10^{-10}\right) \\
.97 \times 10^{-14} \\
.65 \times 10^{-19}\end{array}$ & \begin{tabular}{l}
\multicolumn{1}{c}{ Kaye } \\
$6.716 \times 10$ \\
$6.461 \times 10$ \\
$4.534 \times 10$ \\
$8.450 \times 10$ \\
$7.958 \times 10$ \\
$5.45 \times 10$
\end{tabular} & $\begin{array}{l}0^{-4} \\
0^{-4} \\
0^{-4} \\
0^{-5} \\
0^{-6} \\
0^{-7}\end{array}$ & $\begin{array}{l}\left.6 \times 10^{-8}\right) \\
\left.2 \times 10^{-10}\right) \\
\left.1 \times 10^{-11}\right) \\
\left.1 \times 10^{-15}\right) \\
50 \times 10^{-20}\end{array}$ \\
\hline
\end{tabular}

* As given by Kaye [4], and as calculated in this paper.

$\dagger$ Note: Numbers in parenthesis were derived by extrapolation in Figure 1.

it is seen that $F(n, x)$ approaches 1 as $x$ increases and as $n$ decreases. This is also shown clearly in Figure 2, which gives $1-F(n, x)$ as a function of $x$ with $n$ constant. These figures also permit interpolation and extrapolation to find approximate values for $F(n, x)$ at values of $x$ and $n$ other than those given.

From these results values for $i^{n}$ erfc $x$ were calculated using equation (11) and are given in Table 2 . Since $2 \exp \left(-x^{2}\right) / \sqrt{\pi}(2 x)^{n+1}$ can be calculated very accurately, the final fractional error in $i^{n}$ erfc $x$ is virtually that of $F(n, x)$. For large $x$ the variation in $2 \exp \left(-x^{2}\right) / \sqrt{ } \bar{\pi}(2 x)^{n+1}$ as a function of $x$, however, is very much greater than for $F(n, x)$, so that the final results are quite adequate for engineering applications. This is illustrated in Figure 3, in which $i^{n} \operatorname{erfc} x$ is given as a 


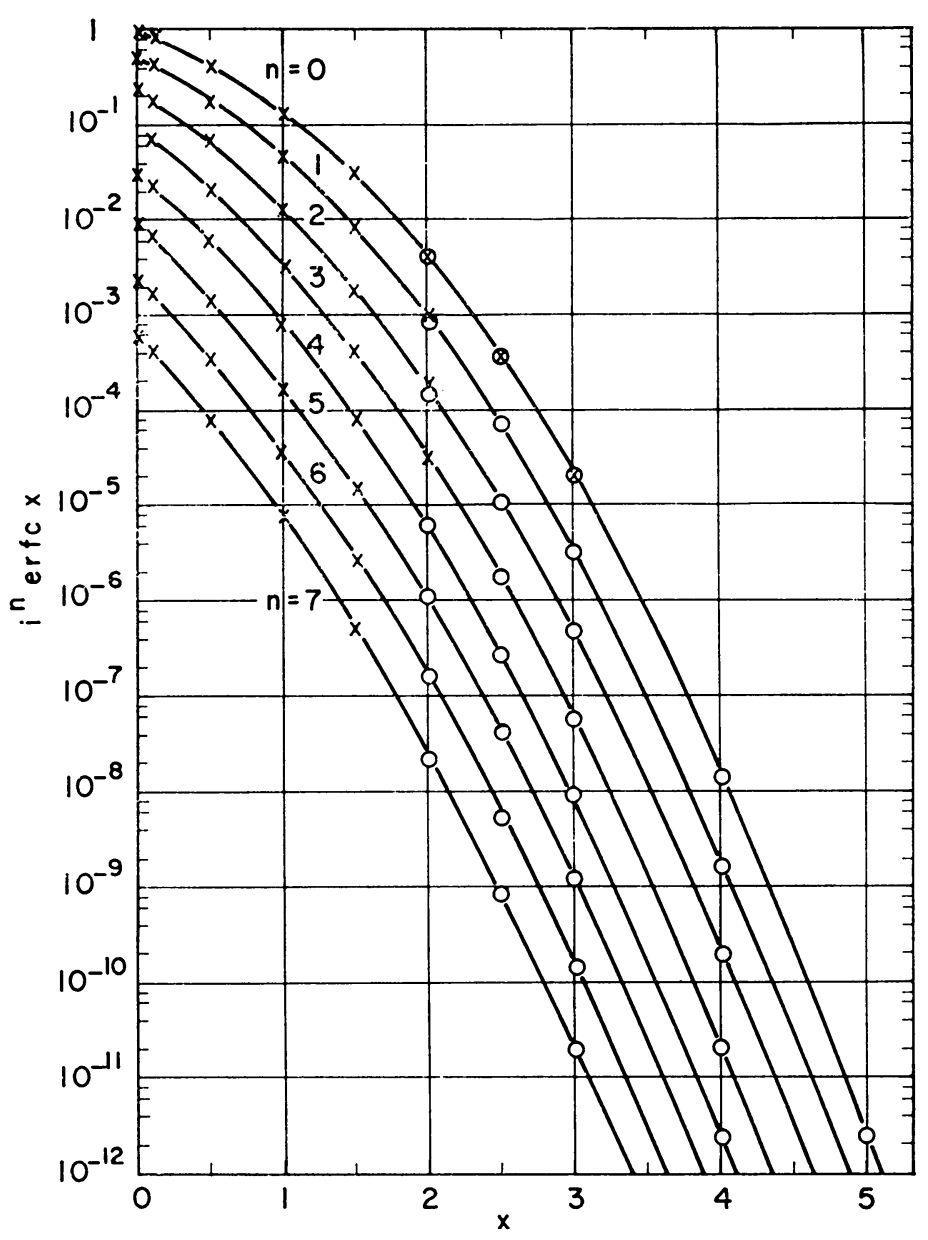

Fig. 3. Repeated Integrals of the Error Function Complement as a Function of $x . x-$ values from Kaye [4]. C-values from this paper.

function of $x$ for various values of $n$. It is apparent that these functions vary so rapidly with $x$ that slight fractional errors become unimportant for engineering applications.

4. Conclusions. New calculation techniques have been derived for the repeated integrals of the error function. These techniques do not depend on previous brute force methods using repeated differences which rapidly run out of significant figures even on large digital computers. Some new values of $i^{n}$ erfc $x$ for large values of $x$ were calculated. These were plotted in such a manner that approximate values can easily be found for all values of $x$.

\section{Materials Sciences Laboratory}

Aerospace Corporation

El Segundo, California

1. D. R. HARTREe, "Some properties and applications of the repeated integrals of the error function," Mem. Manchester Lit. and Phil. Soc., v. 80, 1935, p. 85-102. 
2. H. S. Carslaw \& J. C. JAeger, Conduction of Heat in Solids, second edition, Oxford, 1959 , p. 52, 63, 77, 482-485.

3. JoSEPH KAYE \& V. C. M. YEH, "Design charts for transient temperature distribution resulting from aerodynamic heating at supersonic speeds," $J$. Aeronautical Sciences, v. 22, 1955 , p. 755-763.

4. JosEPH KAYE, "A table of the first eleven repeated integrals of the error function," J. Math. and Physics, v. 34, 1955, p. 119-125.

5. British Association For Advancement Of Science, Mathematical Tables, University Press, Cambridge, 1951, v. I, Table XV.

6. J. Arthur Greenwood \& H. O. Hartley, Editors, Guide to Tables in Mathematical Statistics, Princeton Univ. Press, Princeton, N. J., 1962, Secs. 1.21 and 1.33.

7. A. Fletcher, J. C. P. Miller, L. Rosenhead, \& L. J. Comrie, Editors, An Index to Mathematical Tables, second edition, Addison-Wesley, Reading, Massachusetts, 1962, v. 1. Part 1, Section 15.

8. O. S. Beryland, R. I. Gavrilova \& A. P. Prudnikov, Tables of Integral Error Functions and Hermite Polynomials, Pergamon Press, Oxford, 1962.

9. Nat. Bur. Standards, Tables of the Error Function and Its Derivative, Applied Math. Series No. 41, 1954. 